\title{
Balance Analysis of Microstrip-to-CPS Baluns and Its Effects on Broadband Antenna Performance
}

\author{
Dong Sik Woo, Young-Ki Cho, and Kang Wook Kim \\ The School of Electrical Engineering and Computer Science, Kyungpook National University, Sankyuk-dong Buk-gu, \\ Daegu 702-701, Republic of Korea \\ Correspondence should be addressed to Kang Wook Kim; kang_kim@ee.knu.ac.kr
}

Received 26 October 2012; Revised 11 March 2013; Accepted 12 March 2013

Academic Editor: Zhongxiang Q. Shen

Copyright (C) 2013 Dong Sik Woo et al. This is an open access article distributed under the Creative Commons Attribution License, which permits unrestricted use, distribution, and reproduction in any medium, provided the original work is properly cited.

\begin{abstract}
Amplitude and phase balances of two types of microstrip-(MS-) to-coplanar stripline (CPS) baluns have been analyzed through simulations and measurements, and their effects on broadband antenna performance are investigated. The impedance bandwidth of the balun determined by a back-to-back configuration can sometimes overestimate the balun operating bandwidth. With the conventional balun with a $180^{\circ}$ phase delay line, it is observed that the balun balance over the operating frequencies becomes much more improved as the CPS length increases to over $0.1 \lambda_{g}$. As compared with the conventional balun, the proposed MS-to-CPS balun demonstrated very wideband performance from 5 to over $20 \mathrm{GHz}$. With the proposed balun, amplitude and phase imbalances are within $1 \mathrm{~dB}$ and $\pm 5^{\circ}$, respectively. Effects of the balun imbalance on overall broadband antenna performance are also discussed with a quasi-Yagi antenna and a narrow beamwidth tapered slot antenna (TSA).
\end{abstract}

\section{Introduction}

Planar end-fire antennas have been widely used in microwave/millimeter-wave systems because they exhibit low cost, low weight, low profile, and easy integration with microwave integrated circuits (MICs). For the design of balanced planar antennas, the balun structure as the feed of antennas often determines the overall performance of the antennas. The balun transforms an unbalance transmission line such as microstrip line (MS) into a balanced line such as coplanar stripline (CPS). CPS is a balanced transmission line offering flexibility in the design of planar circuits in series or shunt configuration and is often used in applications such as balanced antennas, uniplanar mixers, and optoelectric devices. Therefore, practical designs of the MS-to-CPS balun (or transition) with good performance have drawn many researchers' attention.

In fact, various microstrip-to-CPS baluns which provide broadband performance have been reported in the literature [1-10]. The transition proposed in [1] required to modify the ground plane and to use a long tapered microstrip line on top layer in order to match the characteristic impedance of CPS. The fabrication of this transition required accurate alignment. Also, the transitions based on the mode conversion $[2,3]$ and coupling method $[4,5]$ were reported. These transitions, however, were only suitable for narrowband applications. Moreover, the transitions were only compatible with high dielectric-constant substrates $\left(\varepsilon_{r}>10\right)$ in order to obtain low characteristic impedance of the CPS, small size, and easy matching to microstrip impedance of $50 \Omega$. Also, a doubleY balun was reported [6], but, to obtain good bandwidth properties, the balun was implemented on a high dielectricconstant substrate with very small gap sizes $(<100 \mu \mathrm{m})$ to minimize the resonance of parasitic even mode. On the other hand, some transitions compatible with low dielectricconstant substrates have been reported $[1,7]$. These transitions, however, used a long and complex ground plane structure to match the high characteristic impedance of CPS $(>100 \Omega)$ to $50 \Omega$. Other various feeding structures such as artificial transmission line based MS-to-CPS transition in [8] and two parallel strips printed on opposite sides of the substrate with truncated ground plane [9] were also reported.

Recently, a novel ultra-wideband MS-to-CPS transition [10] was reported by the authors' group. This balun did not require bulky resonant circuits, and broad impedance bandwidth was inherently achieved by smooth field transformations and good impedance matching through the transition structures. This transition structure has been used for various 
applications due to its excellent in-band return loss and under $1 \mathrm{~dB}$ insertion loss for ultra-wideband frequencies to over $40 \mathrm{GHz}$.

The planar quasi-Yagi antenna was originally introduced by Deal et al. [11]. Due to its wideband performance, compact size, and end-fire characteristics, the quasi-Yagi antenna had immediately attracted a lot of attention. In order to feed this antenna, a uniplanar transition using a 180-degree delay line was used in [3], and this transition had become popular as the feed of quasi-Yagi antennas [11-14]. The transition with a 180degree phase delay line, which generated the odd mode on parallel transmission lines with the truncated ground, often limited the frequency bandwidth. To use this transition as a feed for quasi-Yagi antennas in [11-20], the design of the transition was mostly based on minimizing the insertion loss and maximizing return loss for the operating bandwidth. It is shown, in this paper, that the impedance bandwidth of the transition based on low insertion loss and maximum return loss does not necessarily mean good amplitude and phase balance of the balun.

In this paper, the amplitude and phase balances of the two types of MS-to-CPS transitions have been analyzed, and effects of these baluns on antennas are investigated. Amplitude and phase imbalances of the CPS strips and their effects on antenna radiation performances were not sufficiently described in the previous literature [11-20]. To evaluate the two baluns used as feed networks of quasi-Yagi antennas $[12,21]$, comparison studies are performed. Firstly, the baluns are analyzed in a back-to-back configuration, which is a common way of verifying the transition. Secondly, the reflection coefficient of a single balun terminated in a load [22] is obtained. Next, the amplitude and phase imbalances of two baluns as function of frequency and CPS length are analyzed. Finally, by connecting baluns to a high gain and narrow beamwidth tapered slot antenna, radiation patterns are compared and the effect of the balun on antenna performances discussed.

\section{Two Types of Quasi-Yagi Antennas}

Perspective structures of quasi-Yagi antennas using the conventional (Case I) and proposed (Case II) MS-to-CPS baluns are shown in Figure 1. The most popular and conventional quasi-Yagi antenna (Case I) introduced by Deal et al. in [11] is shown in Figure 1(a). This antenna consists of a microstrip feed, MS-to-CPS balun, and two dipole-elements: one dipole element is a driver dipole fed by CPS and the second strip is a parasitic director. The ground on the bottom plane is truncated and serves as the reflector for the antenna. The balun adopts a quarter-wave long, $35.4 \Omega$ impedance transformer, followed by a symmetric Tee-junction for signal division, and miters for the $90^{\circ}$ microstrip bends. Following the Tee-junction, 180 degree phase difference is achieved by adjusting the delay line length of one branch so that their difference is $\lambda_{g} / 2$, where $\lambda_{g}$ is the guided wavelength at the center frequency. However, in [11], there was no clear description on how the impedance matching and field matching was performed between the coupled microstrip line on truncated ground (or conductor-backed CPS) and CPS feed networks.

Figure 1(b) shows another configuration of the quasiYagi antenna (Case II) with the proposed MS-to-CPS balun previously reported in [21]. There are two major consideration factors for the balun design, that is, smooth field transformation and optimal impedance matching. The field distribution of the microstrip line is gradually transformed to that of the CPS through the ground-shaped balun structure while providing the ground continuity. The bottom conductor trace is optimally tapered for impedance matching. Broadband amplitude and phase balances between CPS strips are achieved by smooth field transformations and impedance taper through the balun structures.

\section{Balun Performance Analysis}

The two MS-to-CPS baluns were designed and evaluated using the full-wave analysis software ANSYS HFSS. The measurement was carried out by using an Anritsu universal test fixture $38801 \mathrm{~K}$ with the maximum frequency up to $40 \mathrm{GHz}$ and an Anritsu 37397C network analyzer. The characteristic impedance of the CPS is usually higher than that of the microstrip line within typical substrate fabrication limits. In Case I, the characteristic impedance of the CPS is about $105 \Omega$ with $0.3 \mathrm{~mm}$ gap $\left(S_{g}\right)$ between CPS strips and $0.3 \mathrm{~mm}$ strip width $\left(W_{\text {cps }}\right)$ using a 25 mil thickness $\mathrm{RT} /$ Duroid6010 $\left(\varepsilon_{r}=10.2, \tan \delta=0.0023\right)$ substrate. It is noted that this balun is usually designed with a high dielectric constant substrate for impedance matching. For Case II, the characteristic impedance of the CPS is about $107 \Omega$ with 5 mil gap $\left(S_{g}\right)$ between CPS strips and 30 mil strip width $\left(W_{\mathrm{cps}}\right)$ using a 20 mil thickness RO4003 $\left(\varepsilon_{r}=3.38, \tan \delta=\right.$ $0.0027)$ substrate. CPS length $\left(L_{\text {cps }}\right)$ for $\lambda_{g} / 4$ is $129 \mathrm{mil}$ and $211 \mathrm{mil}$, respectively, where $\lambda_{g}$ is the guided wavelength of CPS mode at the center frequency (at $10 \mathrm{GHz}$ ). The coplanar stripline (CPS) length is obtained using conformal-mapping techniques [23].

3.1. Balun in a Back-to-Back Configuration. Firstly, as shown in Figure 2(a), symmetric back-to-back configurations of the baluns, which are typical ways of characterizing transitions (or baluns), are simulated and measured. In Figure 2(b), in Case I, the simulated result indicates that the impedance bandwidth for better than $10 \mathrm{~dB}$ return loss is from 6.5 to 11.4 GHz. On the other hand, in Figure 2(c), the simulated and measured results of the Case II balun indicate that the impedance bandwidth of $10 \mathrm{~dB}$ return loss is from 5 to over $20 \mathrm{GHz}$. As can be seen, the simulated results agree closely with measured results, demonstrating broad impedance bandwidth. It is observed that the measured insertion loss per single balun is less than $1 \mathrm{~dB}$. High insertion loss above $20 \mathrm{GHz}$ may be caused by high loss tangent of the substrate and fabrication tolerances.

3.2. A Single Balun Terminated with a Matched Load. Although the back-to-back configuration analysis helps to determine the impedance bandwidth on the basis of insertion 


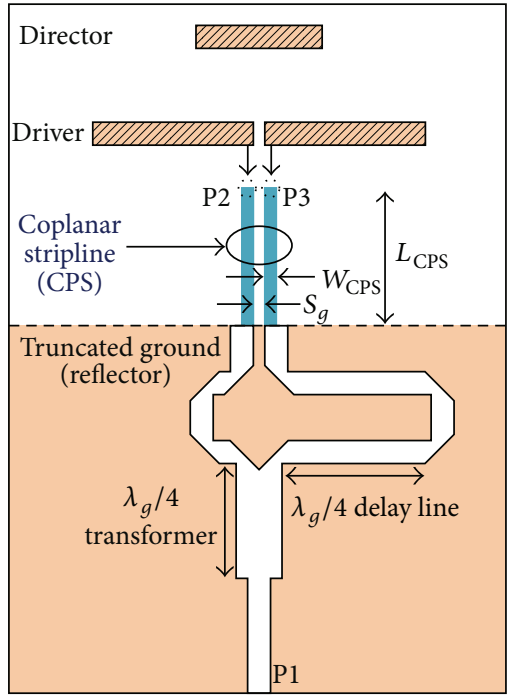

(a) Conventional (Case I)

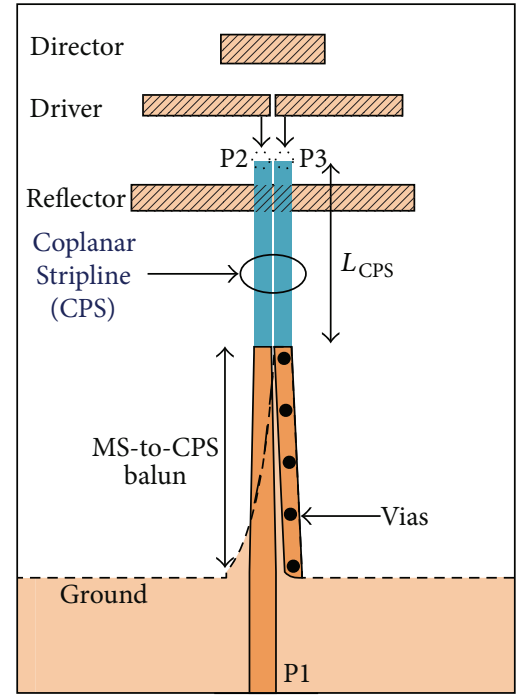

(b) Proposed (Case II)

FIGURE 1: Two types of quasi-Yagi antennas using MS-to-CPS baluns.
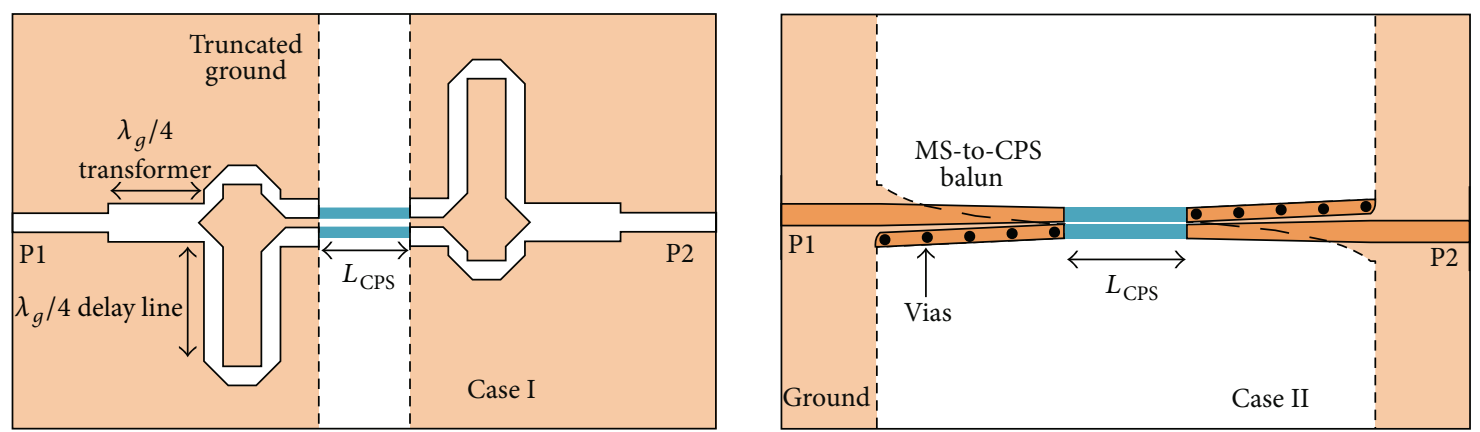

(a) Back-to-back configurations of the MS-to-CPS baluns

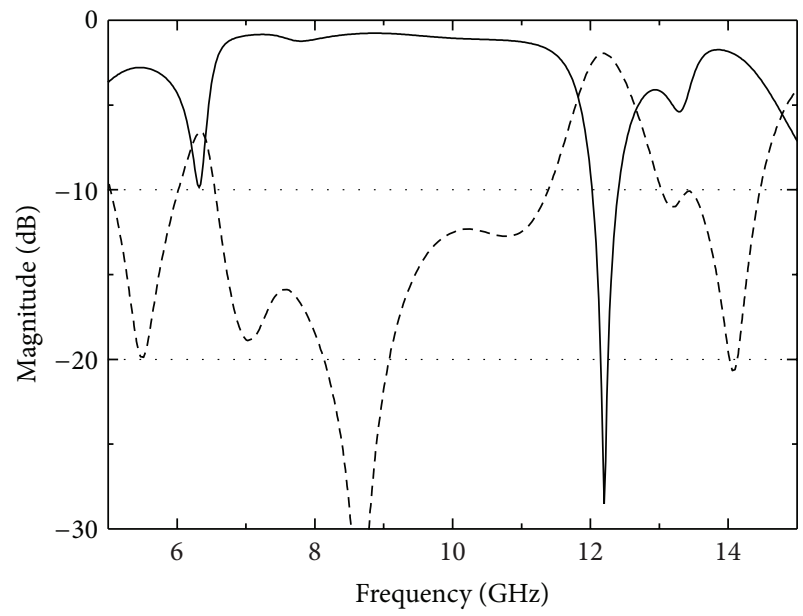

IL (simulated)

- - RL (simulated)

(b) Case I (IL: insertion loss; RL: return loss)

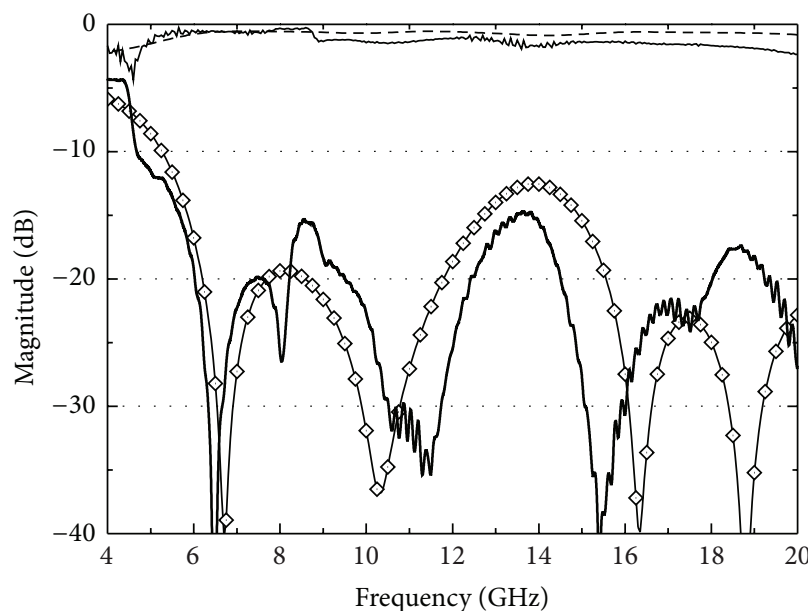

$\begin{array}{ll}-- \text { IL (simulated) } & \diamond \mathrm{RL} \text { (simulated) } \\ \text { - IL (measured) } & \quad \mathrm{RL} \text { (measured) }\end{array}$

(c) Case II (IL: insertion loss; RL: return loss)

FIGURE 2: Back-to-back analysis of the MS-to-CPS baluns. 
loss and return loss, better frequency bandwidth of the balun may be obtained when the CPS output port is terminated with a matched load for a single balun [24]. In HFSS simulation, the CPS output port is terminated with a lumped RLC boundary to get a matched load effect. To obtain an experimental result with the single balun, a surface mount 0402 chip resistor of $110 \Omega$ is used for termination by soldering between two output CPS strips as shown in Figure 3(a).

Simulation and the measurement results for each case are shown in Figures 3(b) and 3(c). In Case I, the measured result indicates that the valid impedance bandwidth for better than $10 \mathrm{~dB}$ return loss is from 6.5 to $12.5 \mathrm{GHz}$. This result is similar with the operation frequency range of the quasi-Yagi antenna in [11]. Next, simulated and measured results of the Case II balun exhibit broad bandwidth of $10 \mathrm{~dB}$ return loss from 4 to over $20 \mathrm{GHz}$. For both cases, the actual usable frequency bandwidths of a single terminated balun are slightly wider than the results of the back-to-back configurations shown in Figure 2. A similar result was also demonstrated in [24]. It can be attributed to the interaction between the two baluns in the back-to-back structure. Therefore, a more practical and accurate usable impedance bandwidth of the balun for balanced antennas can be determined by evaluating a single balun terminated with a matched load [24].

3.3. Analysis of Amplitude and Phase Balances of the Baluns. When designing a balun, however, the actual degree of balances in amplitude and phase between the two output strips of CPS can be more important parameter than impedance bandwidth. Simulation studies have been carried out to investigate on amplitude and phase imbalances at the CPS port. The balun structures for balance analysis are shown in Figure $4(\mathrm{a})$. The CPS length $\left(L_{\mathrm{cps}}\right)$ and transition length $\left(L_{\text {trans }}\right)$ are about $\lambda_{g} / 4$ for each case. Parasitic antenna elements (i.e., driver, director, and reflector) are eliminated. The structure is a three-port network with ports p1, p2, and p3. Similar to the test method suggested by [22], which used the CPS line symmetrically splitting into two CPW ports, the balanced CPS lines symmetrically split into two unbalanced MS output ports. Simple tapered ground planes are used for impedance matching of the two unbalanced MS output ports. In Case I, for mid-band frequency range of 7 to $11 \mathrm{GHz}$, the amplitude difference between the two CPS strips (S21 and S31) is less than $2 \mathrm{~dB}$ as shown in Figure 4(b). Also, with this balun, the phase difference between the two CPS strips is from $171^{\circ}$ to $188^{\circ}$. On the other side, it is observed that variations of amplitude and phase imbalances are nonuniform and changes rapidly near the upper and lower ranges of operating frequencies: amplitude and phase imbalance is abruptly increased to about $6 \mathrm{~dB}$ and $180 \pm 40^{\circ}$, respectively. It is noted that the measured impedance bandwidth of this balun was from 6.5 to $12.5 \mathrm{GHz}$. If a balanced antenna is connected to this balun, the poor excitation of the odd mode in the CPS near upper and lower operating frequency regions may cause significant degradation of the radiation properties.

On the other hand, in Case II, for frequency range of 6.5 to $12.5 \mathrm{GHz}$ as shown in Figure 4(b), the amplitude difference is less than $1 \mathrm{~dB}$. In [22], an amplitude imbalance less than $1.5 \mathrm{~dB}$ with a balun for entire bandwidth was suggested to be adequate for broadband antennas. Also, with the balun, the phase deviation from $180^{\circ}$ between the two CPS strips is maintained within $6^{\circ}$ (from $178^{\circ}$ to $184^{\circ}$ ) over whole operating frequencies. The maximum allowable phase imbalance may be dependent on specific applications; in general, $\pm 5^{\circ}$ deviation from $180^{\circ}$ is considered as a criterion of a well-designed balun [22]. Therefore, the proposed MSto-CPS balun is considered to be sufficiently suitable for the design of broadband balanced antennas such as quasi-Yagi antenna [21], bow-tie antenna [25], and tapered slot antenna [26].

From the results of balun balance analysis, it is noted that the useable and valid operation bandwidth of the balun can be better determined by verifying amplitude and phase balance analysis as function of frequency. Just a simple characterization of impedance bandwidth with a back-toback configuration or with a load-terminated CPS may not be sufficient to evaluate the balun performance.

Another balance analysis of the balun is performed by changing the CPS length $\left(L_{\mathrm{cps}}\right)$ up to quarter wavelength $\left(\lambda_{g} / 4: 129 \mathrm{mil}\right.$ for Case I and $211 \mathrm{mil}$ Case II). In Case I, as shown in Figure 5(a), it is interesting to observe that the amplitude imbalances are decreased with increase of the CPS length, that is, typically from $4 \mathrm{~dB}$ to less than $2 \mathrm{~dB}$ as $L_{\text {cps }}$ increases up to $0.25 \lambda_{g}$. For mid-band frequency range of 7 to $11 \mathrm{GHz}$, the phase imbalances are also significantly reduced with increase of the CPS length $\left(171^{\circ}\right.$ to $\left.188^{\circ}\right)$. With a short CPS length, the $180^{\circ}$ phase delay path excites odd mode only near the center frequency, and the amplitude and phase imbalances are significant at other frequencies. As the CPS length increases $\left(>0.1 \lambda_{g}\right)$, the amplitude and phase imbalances over the operating frequency range tend to be much reduced due to increased odd mode conversion. However, at upper and lower frequencies of the impedance bandwidth of the balun, amplitude and phase imbalances are more significant as compared with the mid-band frequencies. It can be attributed to the mismatch and discontinuity between the two transmission lines of conductor backed coupled microstrip and CPS. Especially, in Case I, mode mismatches and radiation fields are observed by simulation studies. However, in Case II, variations of amplitude and phase imbalances are kept very small and uniform for whole operation frequencies as shown in Figure 5(b). Typically, amplitude imbalances are maintained less than $1 \mathrm{~dB}$ as $L_{\text {cps }}$ increases up to $0.25 \lambda_{g}$, and the phase imbalances are kept from $178^{\circ}$ to $184^{\circ}$ for the full operating bandwidth.

In design of the quasi-Yagi antenna based on Yagi-Uda theory, in general, the optimum length of $L_{\text {cps }}$ is approximately $\lambda_{g} / 4$. With the Case I balun, the truncated ground plane is normally used as a reflector optimized for good return loss and low backside radiation pattern. Therefore, in practical quasi-Yagi antenna design, $L_{\mathrm{cps}} \sim \lambda_{g} / 4$ is usually an adequate CPS length. With Case II, however, since the CPS length does not affect the amplitude and phase balance, there is a freedom in choosing the appropriate CPS length for specific applications. 


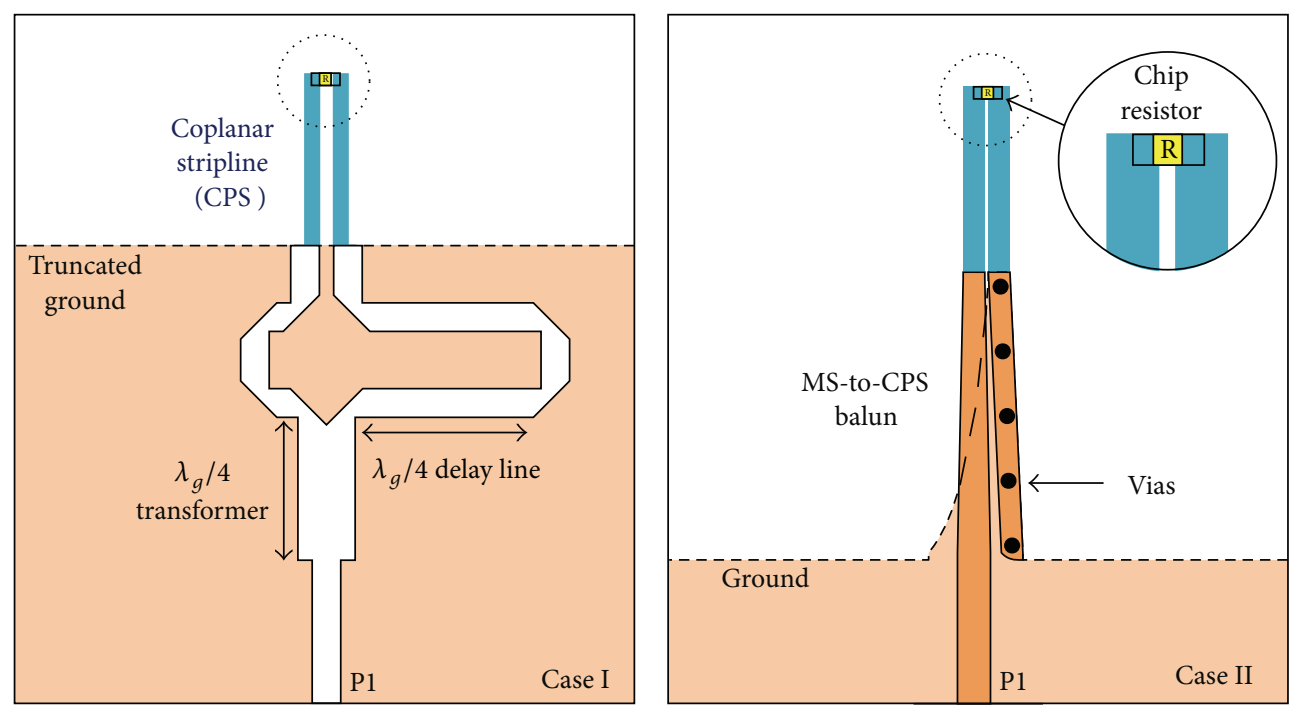

(a) Test layouts

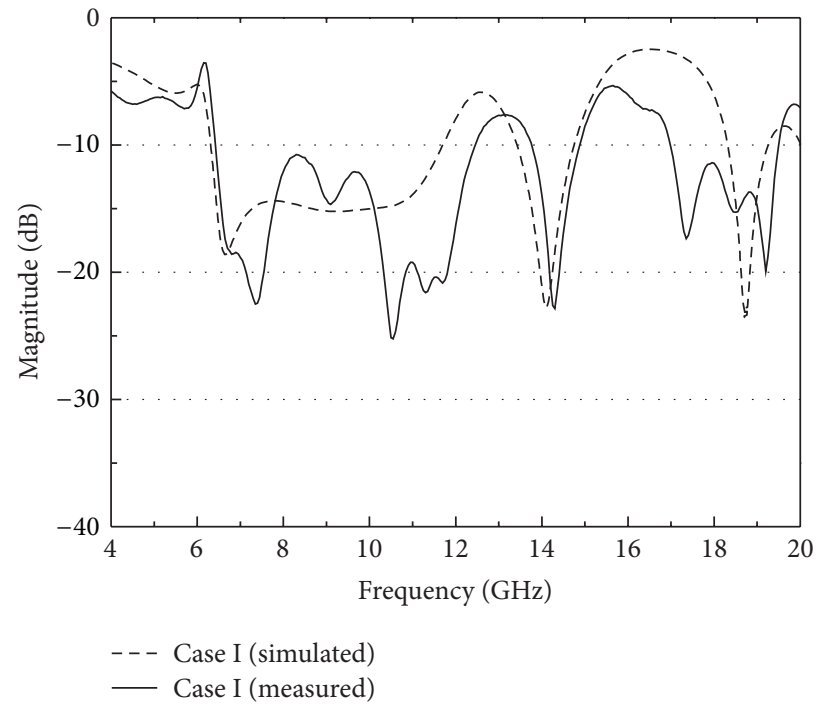

(b) Simulated and measured return loss (Case I)

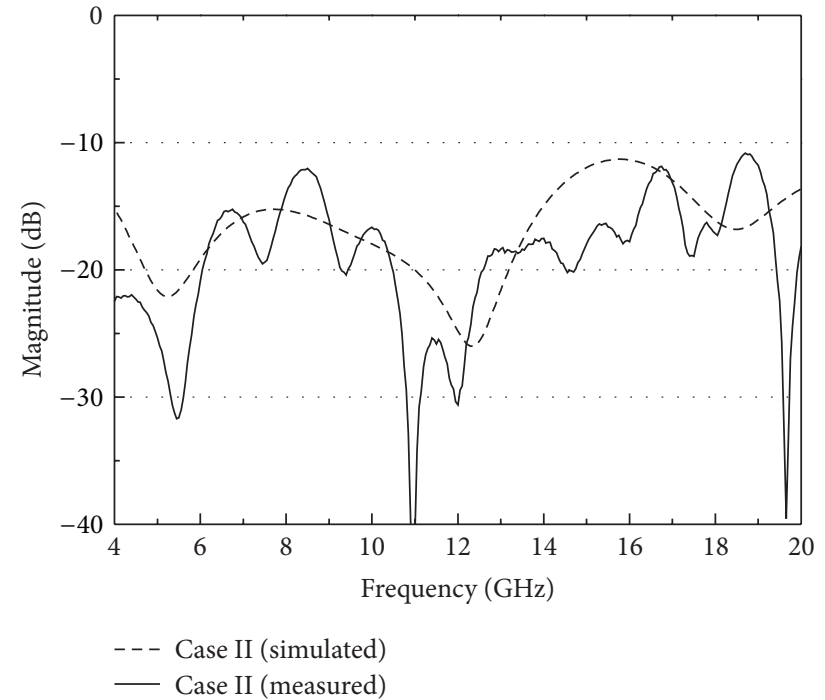

(c) Simulated and measured return loss (Case II)

FIgURE 3: MS-to-CPS baluns terminated with a matched load.

\section{Balun Imbalance Effects on Antenna Performance}

In order to investigate effects of the balun imbalance on the performance of antennas, the two types of baluns were connected to two types of antennas (quasi-Yagi and tapered slot antennas), and the corresponding radiation characteristics (gain, sidelobe, and angle deviation of the maximum gain direction) were obtained through simulation.

For quasi-Yagi antennas due to their wide beam widths, it is difficult to obviously observe antenna gain variations and angle deviation of the maximum gain direction if the bandwidth of the antenna is much less than that of the balun. The impedance bandwidths of the Case I balun and conventional quasi-Yagi antennas were usually less than 50\% (7.2-12 GHz). Typically, quasi-Yagi antennas using the Case I balun were demonstrated as broadband antennas (40\%-50\% for VSWR $<2)$ or as narrow bandwidth antennas $(10 \%-20 \%$ for VSWR <2) with high gain performance [11]. Therefore, as long as the antenna bandwidth is well within the bandwidth of the balun, performances of the quasi-Yagi antennas will not be strongly influenced by the amplitude and phase imbalances. These results are summarized in Table 1.

On the other hand, by adjusting the size and gap of the antenna elements, the bandwidth of quasi-Yagi antennas (without the balun) can be widened up to $70 \%$. In this case, the bandwidth of the quasi-Yagi antenna is wider than that of the balun, and, when it is connected to the balun, balun imbalance effects are certainly observed in radiation patterns and gain variations near the lower and upper ranges of the balun impedance bandwidth. With the Case II balun, however, the bandwidth of the balun is always much wider than 

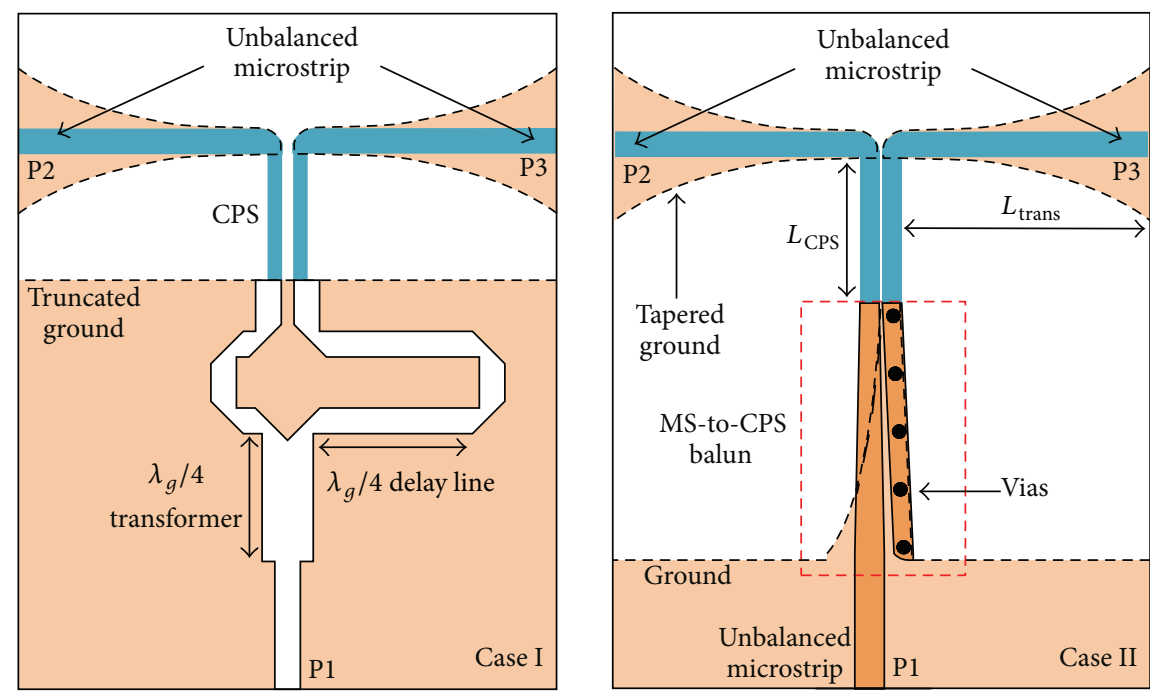

(a) Test layouts

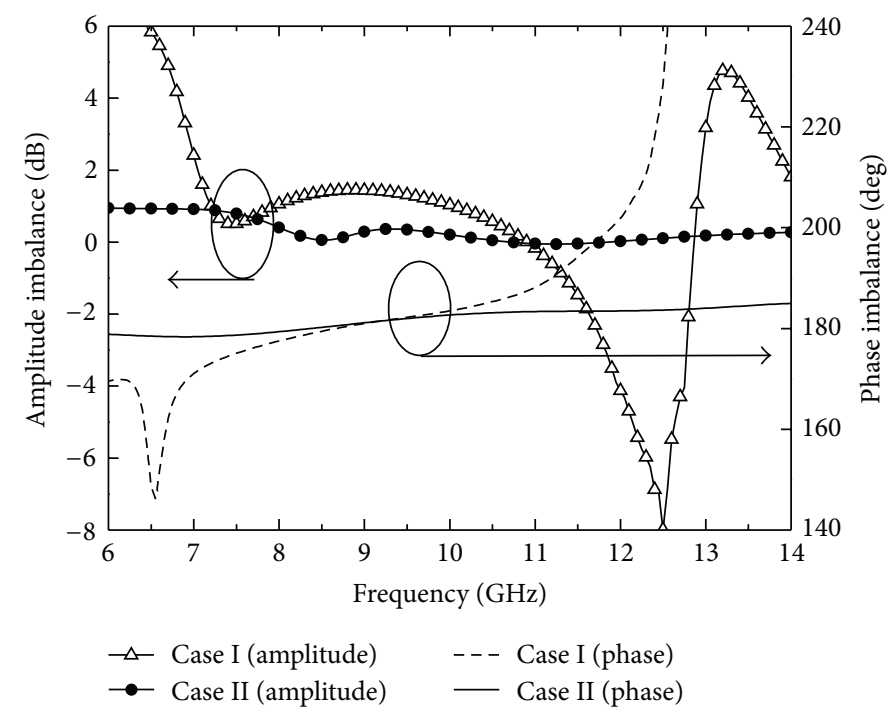

(b) Amplitude and phase imbalances (simulation)

FIGURE 4: Balance analysis of MS-to-CPS baluns.

those of quasi-Yagi antennas, and the uniform radiation patterns are obtained for the whole operating frequency ranges.

To clearly demonstrate radiation pattern degradations due to the balun imbalance, a broadband and narrow beamwidth TSA was connected to the balun. In this case, the antenna bandwidth is much wider than that of the Case I balun, and the performance was analyzed for the balun impedance bandwidth in the frequency range from 6.5 to 12.5 GHz as shown in Figure 6. Each TSA was designed by following guidelines in [26] for whole X-band operating frequencies. Two TSAs were optimized using different substrates with length of $3 \lambda_{0}$ and gain of $12 \mathrm{dBi}$. In Case I, in order to eliminate the substrate thickness effect of TSA using high dielectric constant substrate, thickness of the TSA section was reduced to $10 \mathrm{mil}$ to generate well-behaved travelling waves in the antenna [27]. Figures 6(a) and 6(b) show the simulated E-plane radiation patterns of the antennas for the balun impedance bandwidth. It is observed that the simulated antenna gain varies from 8.3 to 12.7 (Case I) and 10.7 to $13 \mathrm{dBi}$ (Case II) for frequency range of 6.5 to $12.5 \mathrm{GHz}$. As can be seen, the gain variations and sidelobe levels of the Case I antenna are much higher than those of the Case II antenna. In addition, the end-fire directions or the maximum gain angles of the Case I antenna are somewhat shifted at upper and lower frequencies of 6.5 and $12.5 \mathrm{GHz}$. The nonuniform and poor radiation patterns are caused by poor phase balance of the balun even within the impedance bandwidth. With the Case II antenna, however, the radiation patterns are much more uniform due to better amplitude and phase balance of the feeding network.

The effects of the balun imbalances for both antenna configurations are summarized and compared in Table 1. As can 


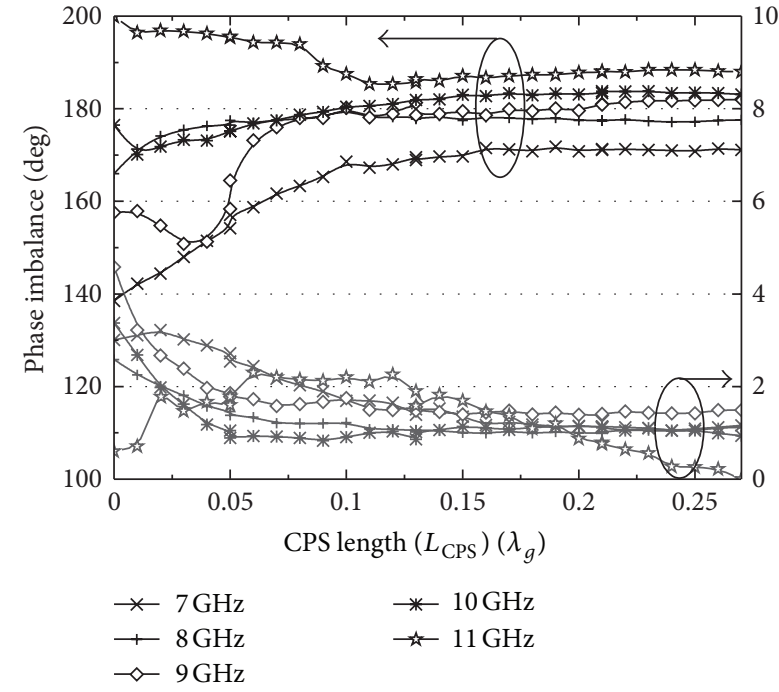

(a) Case I

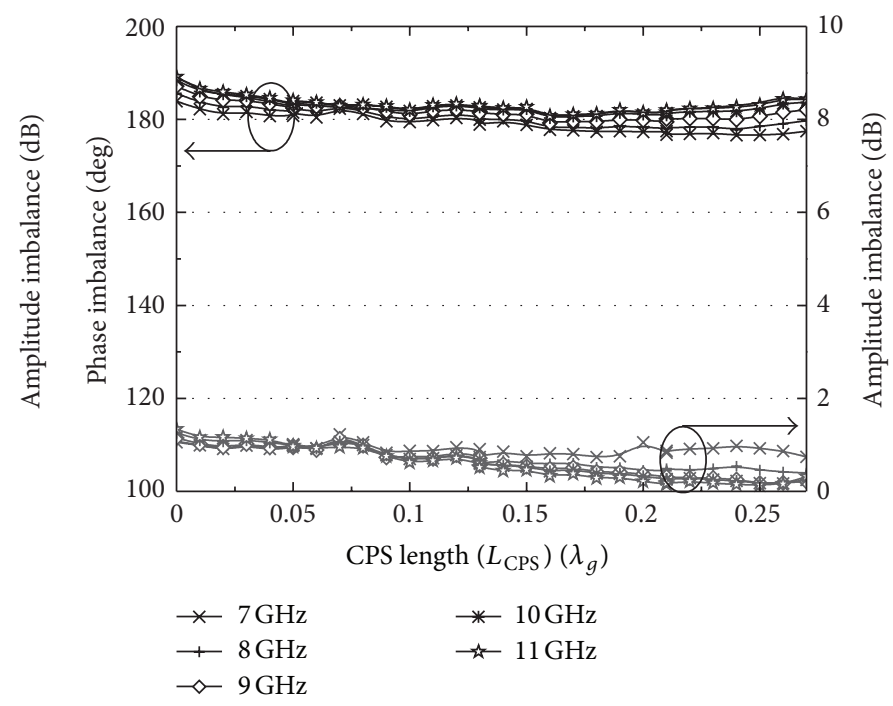

(b) Case II

FIgURE 5: Amplitude and phase imbalances as a function of CPS length.

TABle 1: Performance summary of the balance analysis.

\begin{tabular}{|c|c|c|c|c|}
\hline Parameters & Unit & Remarks & $\begin{array}{c}\text { Conventional structure [11] } \\
\text { (Case I) }\end{array}$ & $\begin{array}{c}\text { Proposed structure } \\
\text { (Case II) }\end{array}$ \\
\hline \multirow{2}{*}{ Balun operating bandwidth } & \multirow{2}{*}{$\mathrm{GHz}$} & Back-to-back & $6.5-11.4$ & $5-20$ \\
\hline & & single terminated & $6.5-12.5$ & $4-20$ \\
\hline \multirow{2}{*}{ Amplitude imbalance } & \multirow{2}{*}{$\mathrm{dB}$} & 7-11 GHz. & $< \pm 2$ & $< \pm 0.5$ \\
\hline & & $6.5-12.5 \mathrm{GHz}$ & $< \pm 6$ & \\
\hline \multirow{2}{*}{ Phase imbalance } & \multirow{2}{*}{ degree } & 7-11 GHz. & $< \pm 8$ & $< \pm 3$ \\
\hline & & $6.5-12.5 \mathrm{GHz}$ & $< \pm 20$ & \\
\hline \multirow{2}{*}{ Antenna operating bandwidth } & \multirow{2}{*}{$\mathrm{GHz}$} & Quasi-Yagi & $8-12$ & $8-12$ \\
\hline & & TSA & $6.5-12.5$ & $6.5-12.5$ \\
\hline \multirow{2}{*}{ Antenna gain } & \multirow{2}{*}{$\mathrm{dBi}$} & Quasi-yagi & $3.5-5.5$ & $4.9-6.3$ \\
\hline & & TSA & $8.3-12.7$ & $10.7-13$ \\
\hline \multirow{2}{*}{ Angle deviation (max. gain direction) } & \multirow{2}{*}{ Degree } & Quasi-yagi & \pm 4 & \pm 4 \\
\hline & & TSA & \pm 7 & \pm 2 \\
\hline Efficiency & $\%$ & Quasi-yagi & $92-96$ & $93-96$ \\
\hline \multirow{2}{*}{ Occupied size } & \multirow{2}{*}{$\mathrm{mm}$} & Quasi-yagi & $13 \times 20$ & $20 \times 30$ \\
\hline & & TSA & $33 \times 116$ & $36 \times 123$ \\
\hline Substrate material & & & $\begin{array}{c}\text { Duroid6010 }\left(\varepsilon_{r}=10.2\right) \\
t=0.762 \mathrm{~mm}\end{array}$ & $\begin{array}{c}\mathrm{RO} 4003\left(\varepsilon_{r}=3.38\right) \\
t=0.508 \mathrm{~mm}\end{array}$ \\
\hline
\end{tabular}

be seen, the antenna adopting the proposed balun exhibits much wider bandwidth and uniform radiation performances as compared with the antenna using the Case I balun. From these results, it has been demonstrated that the proposed MS-to-CPS balun is well balanced in amplitude and phase and is suitable for all applications of broadband balanced antenna. Therefore, to minimize the balun imbalance effects on the antenna performance, especially when the bandwidths of broadband balanced antennas are comparable to or wider than that of the feeding balun, amplitude and phase balance analysis may be critical.

\section{Conclusion}

In this paper, amplitude and phase balances for the two types of MS-to-CPS baluns have been analyzed, and their effects on antenna performance for X-band end-fire antennas are 

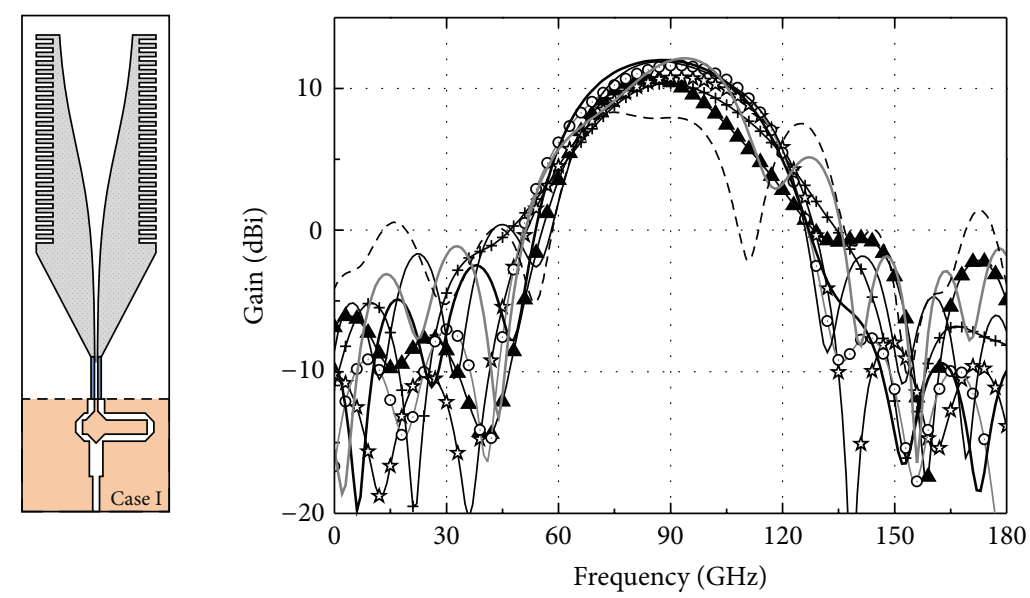

(a) Case I
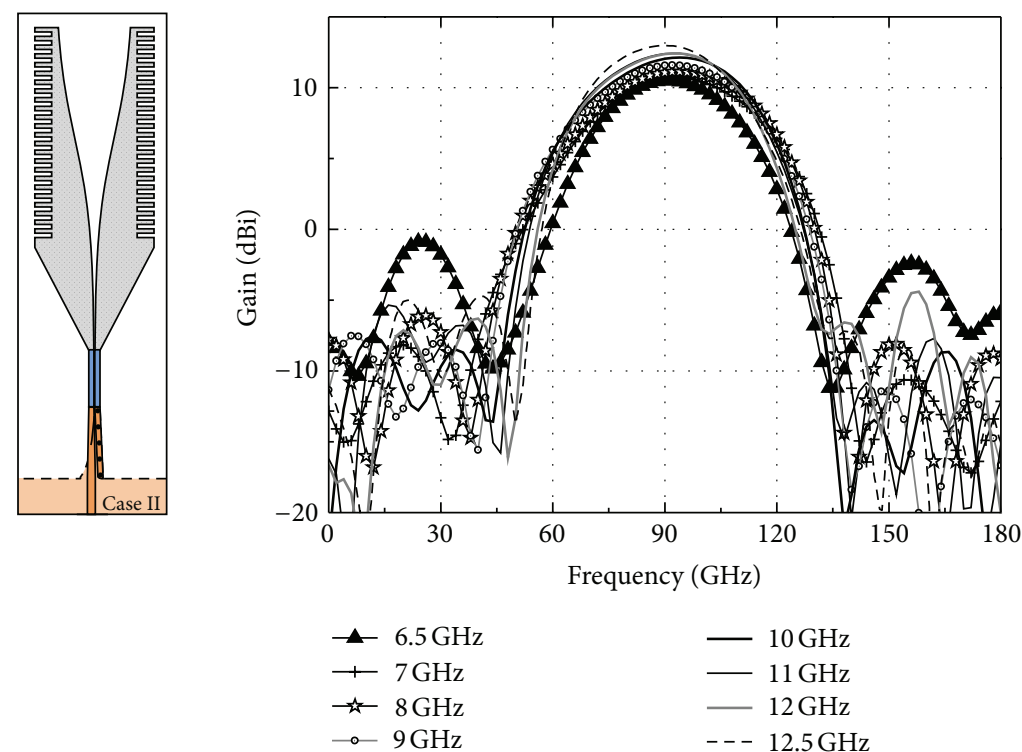

(b) Case II

FIGURE 6: E-plane radiation patterns of TSAs (simulation).

investigated. The impedance bandwidths of the baluns have been obtained through a back-to-back configuration and a single balun terminated with a load. To determine the valid operating frequency range of the balun, amplitude and phase imbalances of baluns with changing the CPS length have been evaluated. For the conventional balun structure with a $180^{\circ}$ phase delay line, the valid operating bandwidth can be much less than the impedance bandwidth, while the proposed balun demonstrated a very wide bandwidth valid to over $20 \mathrm{GHz}$. Finally, to evaluate the effects of balun imbalance, radiation patterns of a broadband and high gain TSA are simulated and compared. For broadband antennas with bandwidth comparable to or wider than that of the feeding balun, radiation performance can be degraded near the lower and upper ranges of the balun impedance bandwidth. From these results, it is shown that the analysis of amplitude and phase imbalances of the balun is a very effective way to determine the valid bandwidth of the balun and the performance of broadband balanced antennas. The proposed ultra-wideband MS-to-CPS balun may be applicable to a variety of broadband microwave/mm-wave phased arrays and imaging systems.

\section{Acknowledgment}

This research was supported by the The Ministry of Knowledge Economy (MKE), Republic of Korea, under the Convergence Information Technology Research Center (CITRC) support program (NIPA-2012-H0401-12-1006) supervised by the National IT Industry Promotion Agency (NIPA).

\section{References}

[1] Y. H. Suh and K. Chang, "A wideband coplanar stripline to microstrip transition," IEEE Microwave and Wireless Components Letters, vol. 11, no. 1, pp. 28-29, 2001. 
[2] N. I. Dib, R. N. Simons, and L. P. B. Katehi, "New uniplanar transition for circuit and antenna applications," IEEE Transactions on Microwave Theory and Techniques, vol. 43, no. 12, pp. 28682873, 1995.

[3] Y. Qian and T. Itoh, "Broadband uniplanar microstrip-toCPS transition," in Proceedings of the Asia-Pacific Microwave Conference Digest, pp. 609-612, December 1997.

[4] W. H. Tu and K. Chang, "Wide-band microstrip-to-coplanar stripline/slotline transitions," IEEE Transactions on Microwave Theory and Techniques, vol. 54, no. 3, pp. 1084-1088, 2006.

[5] R. N. Simons, N. I. Dib, and L. P. B. Katehi, "Coplanar stripline to microstrip transition," Electronics Letters, vol. 31, no. 20, pp. 1725-1726, 1995.

[6] J. Venkatesan, "Novel version of the double-Y balun: microstrip to coplanar strip transition," IEEE Antennas and Wireless Propagation Letters, vol. 5, no. 1, pp. 172-174, 2006.

[7] T. Chiu and Y. S. Shen, "A broadband transition between microstrip and coplanar stripline," IEEE Microwave and Wireless Components Letters, vol. 13, no. 2, pp. 66-68, 2003.

[8] T. G. Ma, C. W. Wang, R. C. Hua, and J. W. Tsai, "A modified quasi-yagi antenna with a new compact microstrip-to-coplanar strip transition using artificial transmission lines," IEEE Transactions on Antennas and Propagation, vol. 57, no. 8, pp. 24692474, 2009.

[9] G. Zheng, A. A. Kishk, A. W. Glisson, and A. B. Yakovlev, "Simplified feed for modified printed Yagi antenna," Electronics Letters, vol. 40, no. 8, pp. 464-466, 2004.

[10] Y. G. Kim, D. S. Woo, K. W. Kim, and Y. K. Cho, "A new ultrawideband microstrip-to-CPS transition," in Proceedings of IEEE MTT-S International Microwave Symposium Digest, pp. 15631566, June 2007.

[11] W. R. Deal, N. Kaneda, J. Sor, Y. Qian, and T. Itoh, "A new quasi-yagi antenna for planar active antenna arrays," IEEE Transactions on Microwave Theory and Techniques, vol. 48, no. 6, pp. 910-918, 2000.

[12] N. Kaneda, W. R. Deal, Y. Qian, R. Waterhouse, and T. Itoh, "A broad-band planar quasi-Yagi antenna," IEEE Transactions on Antennas and Propagation, vol. 50, no. 8, pp. 1158-1160, 2002.

[13] P. R. Grajek, B. Schoenlinner, and G. M. Rebeiz, "A 24GHz high-gain Yagi-Uda antenna array," IEEE Transactions on Antennas and Propagation, vol. 52, no. 5, pp. 1257-1261, 2004.

[14] N. Nikolic and A. R. Weily, "Compact E-band planar quasi-Yagi antenna with folded dipole driver," IET Microwaves, Antennas and Propagation, vol. 4, no. 11, pp. 1728-1734, 2010.

[15] B. H. Sun, S. G. Zhou, Y. F. Wei, and Q. Z. Liu, "Modified two-element Yagi-uda antenna with tunable beams," Progress in Electromagnetics Research, vol. 100, pp. 175-187, 2010.

[16] D. Wu, Y. Fan, M. Zhao, and Y. Zhang, "Millimeter wave omnidirectional quasi-Yagi array," Progress in Electromagnetics Research Letters, vol. 5, pp. 123-130, 2008.

[17] X. C. Zhang, J. Liang, and J. W. Xie, “The Quasi-Yagi antenna subarray fed by an orthogonal T junction," Progress in Electromagnetics Research Letters, vol. 4, pp. 109-112, 2008.

[18] S. C. Zhao, B. Z. Wang, and W. Shao, "Reconfigurable Yagi-Uda substrate for radar cross section reduction of patch antenna," Progress in Electromagnetics Research B, vol. 11, pp. 173-187, 2009.

[19] M. Bemani and S. Nikmehr, "A novel Wide-Band microstrip Yagi-Uda array antenna for WLAN applications," Progress in Electromagnetics Research B, no. 16, pp. 389-406, 2009.
[20] R. Bayderkhani and H. R. Hassani, "Wideband and low sidelobe linear series fed yagi-like antenna array," Progress in Electromagnetics Research B, no. 17, pp. 153-167, 2009.

[21] D. S. Woo, Y. G. Kim, K. W. Kim, and Y. K. Cho, "Design of quasi-Yagi antennas using an ultra-wideband balun," Microwave and Optical Technology Letters, vol. 50, no. 8, pp. 2068-2071, 2008.

[22] B. Jokanovic, V. Trifunovic, and B. Reljic, "Balance measurements in double-Y baluns," IEE Proceedings, vol. 149, no. 6, pp. 257-260, 2002.

[23] G. Ghione and C. Naldi, "Analytical formulas for coplanar lines in hybrid and monolithic MICs," Electronics Letters, vol. 20, no. 4, pp. 179-181, 1984.

[24] J. Thaysen, K. B. Jakobsen, and J. Appel-Hansen, "A wideband balun-how does it work?" Applied Microwave and Wireless, vol. 12, no. 10, pp. 40-50, 2000.

[25] D. S. Woo, Y. G. Kim, I. B. Kim, Y. K. Cho, and K. W. Kim, "Broadband antennas using a planar Ultra-wideband balun," in Proceedings of 11th IEEE International Conference on Communication Technology (ICCT'08), pp. 305-308, November 2008.

[26] D. S. Woo, Y. G. Kim, Y. K. Cho, and K. W. Kim, "Ultra-wideband Fermi antenna using microstrip-to-CPS balun," IEICE Transactions on Communications, vol. E93-B, no. 8, pp. 22192222, 2010

[27] K. S. Yngvesson, "Endfire tapered slot antennas on dielectric substrates," IEEE Transactions on Antennas and Propagation, vol. 33, pp. 1392-1400, 1985. 

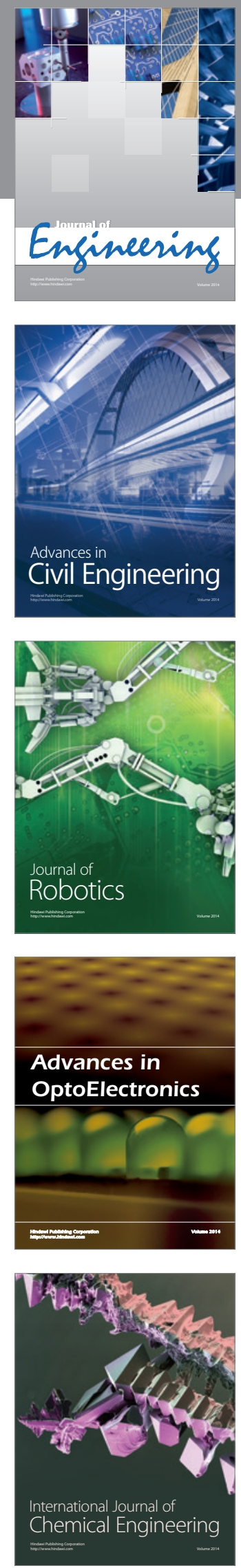

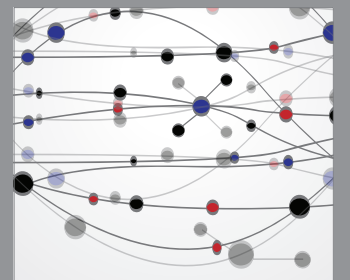

The Scientific World Journal
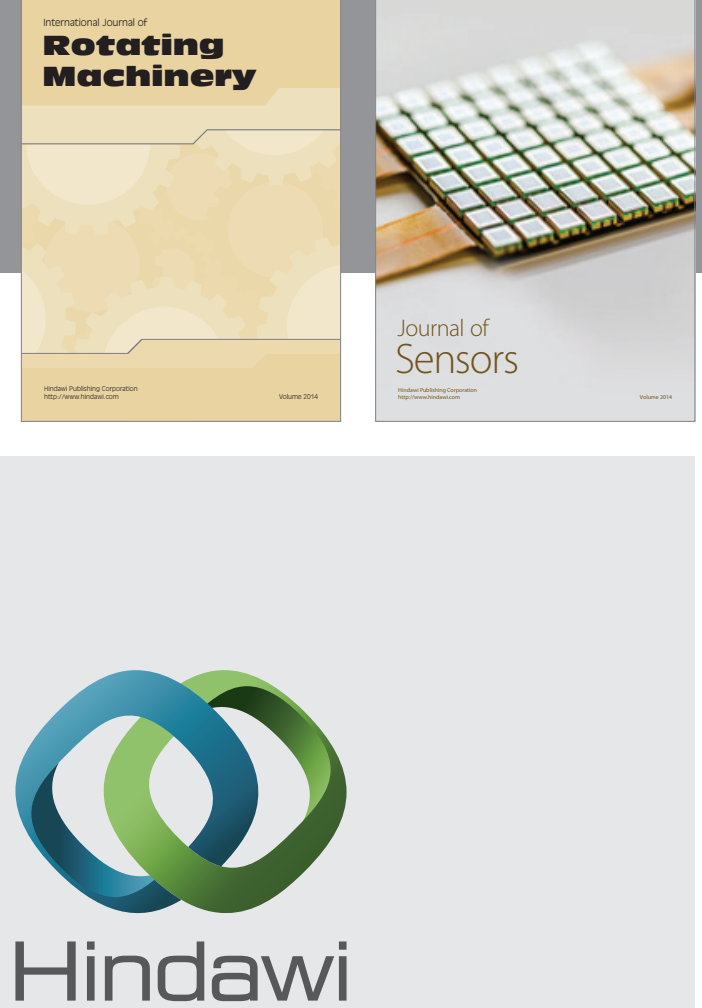

Submit your manuscripts at http://www.hindawi.com
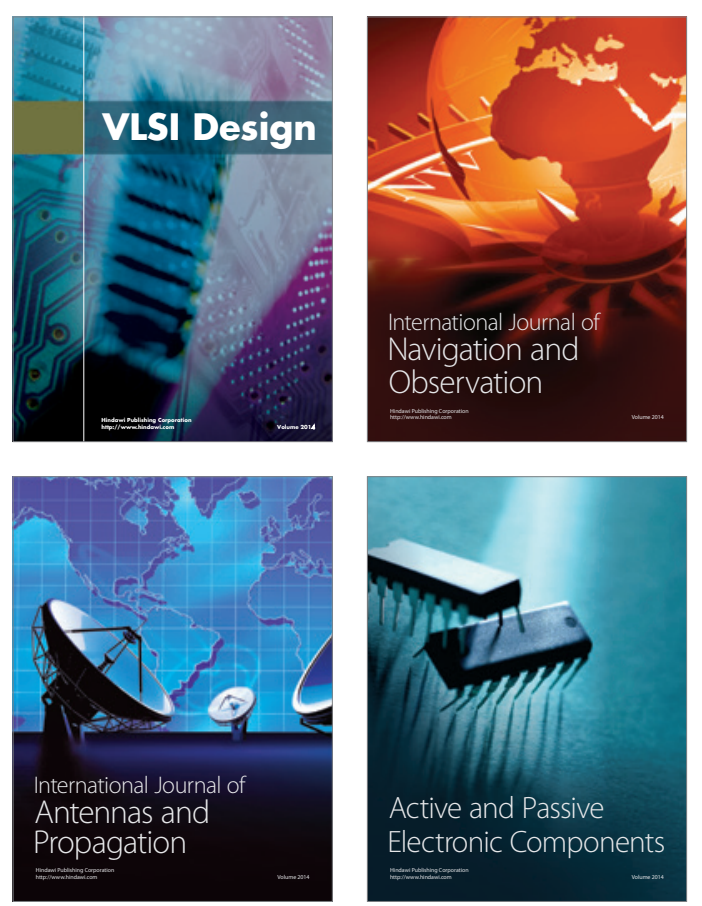
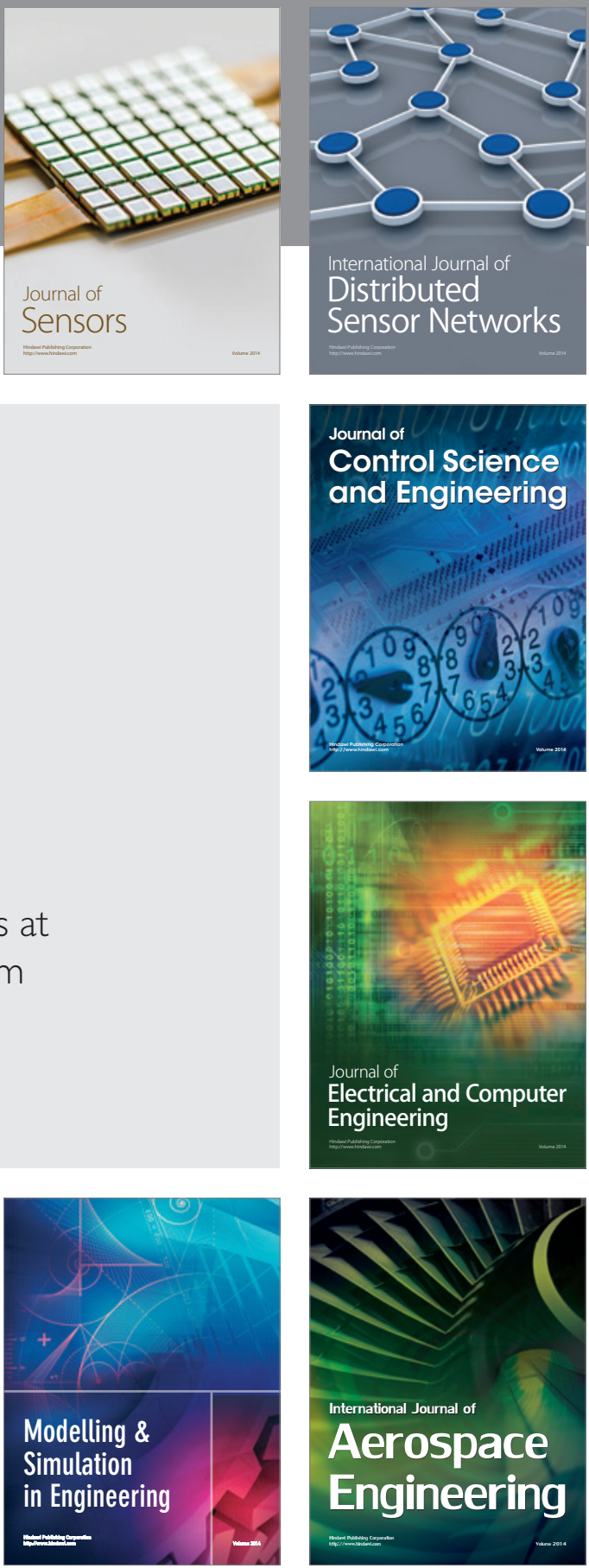

Journal of

Control Science

and Engineering
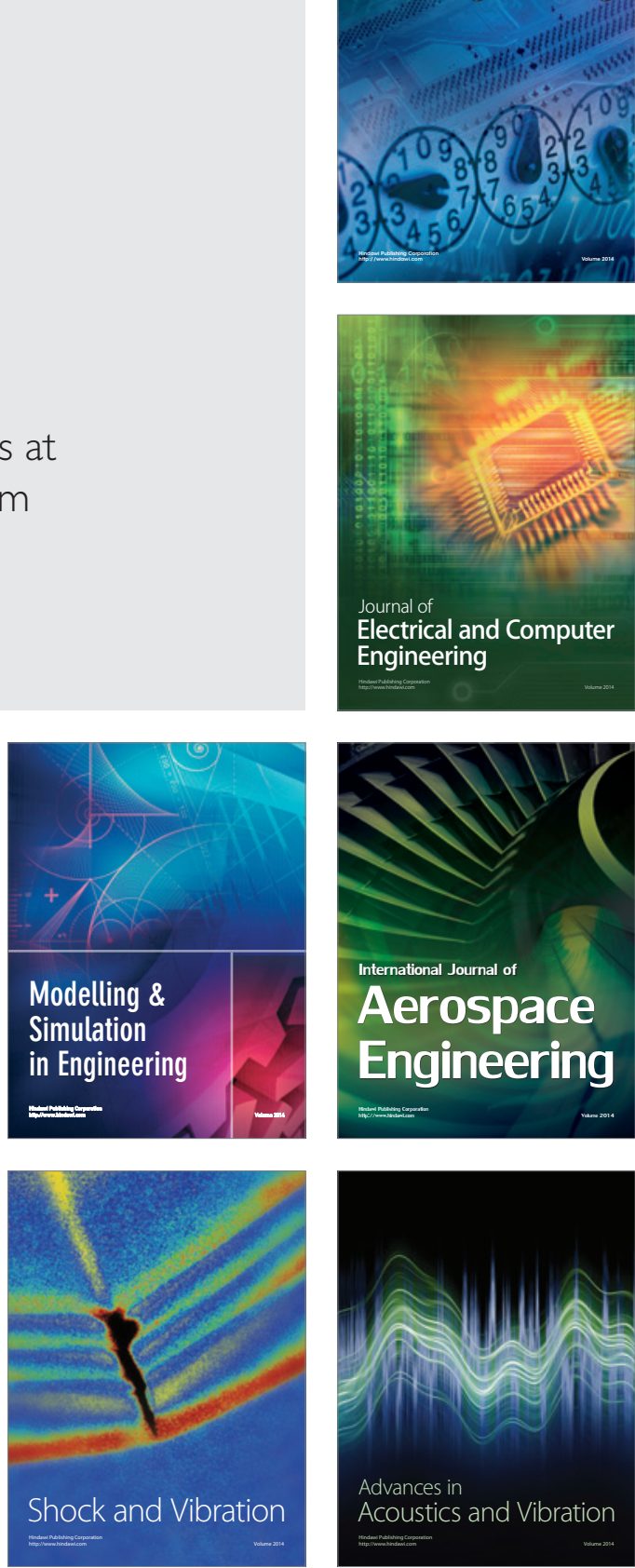